\title{
Palpable Masses Completion Status
}

National Cancer Institute

\section{Source}

National Cancer Institute. Palpable Masses Completion Status. NCI Thesaurus. Code C119916.

A term used to describe the state or condition of the completeness of the palpable masses data. 